\title{
A Model of Isolated, Autologously Hemoperfused Porcine Slaughterhouse Lungs
}

Peter C. Kamusella ${ }^{1,2}$, Christian Wissgott ${ }^{1}$, Christian Grosse-Siestrup ${ }^{2}$, Sven Dittrich ${ }^{2}$, Olaf Hegemann ${ }^{2}$, Dimitri Koios ${ }^{3}$, Juliane von Massenbach ${ }^{3}$, Michael Meissler ${ }^{3}$, Volker Unger ${ }^{3}$, David Quarcoo ${ }^{3 *}$ and David A. Groneberg ${ }^{3 *}$

${ }^{1}$ Dept. of Radiology, Westküstenkliniken Heide, Germany; ${ }^{2}$ Dept. of Comparative Medicine and Experimental Animal Sciences, and; ${ }^{3}$ Institute of Occupational Medicine, Charité - Universitätsmedizin Berlin, Free University and Humboldt University, Germany

\section{Summary}

Introduction: Models of isolated and perfused lungs study pathophysiological phenomena of the airways, but are limited by restricted resemblance to the human situation, non-physiological perfusates or the need for the use of high numbers of laboratory animals. The present model was established to address these difficulties.

Objectives: Aim of the current study was the establishment of an animal model that uses slaughterhouse animals and closely resembles physiological conditions found in humans.

Methods: We used a model of hemoperfused isolated porcine slaughterhouse lungs using autologous blood, metabolically controlled via a dialysis system. Over a period of 135 minutes positive inspiratory pressure, pulmonary arterial pressure, pulmonary vein oxygen partial pressure and lung weight were assessed.

Results: Stable organ function was maintained over 135 minutes with an amount of 2,500-3,000 ml perfusate without fall in pulmonary arterial pressure. During the time the positive inspiratory pressure and lung weight increased, while pulmonary vein oxygen partial pressure decreased.

Conclusions: The present model of isolated hemoperfused slaughterhouse lungs displays a useful new and economic approach to evaluate pulmonary function and toxicity of different substances on an organ level. As a major economic advantage in comparison to models using laboratory animals, the current model might be run using blood and organs obtained from slaughterhouse animals.

Keywords: isolated lung, autologous blood perfusion, pig, slaughterhouse

\section{Introduction}

Perfused isolated animal lung models have been developed to understand pathophysiological events occurring in the airways (Fehrenbach et al., 2001; Schneuwly et al., 1999; Wright et al., 2000). These models have enabled studies of the lung as an intact organ with its physiological intercellular contacts and tissues. The advantage as compared to cell cultures or lung slices is the utilization of the entire organ resulting in a better comparability to the in vivo situation. Additionally, the ex vivo aspect of the model proved to be beneficial, because influencing variables can be defined and the influence of systemic factors might be controlled (Kimose et al., 1990; Samaja et al., 1990). The models have served as a tool to advance existing therapeutical concepts and develop new treatment options for lung diseases. They are especially useful in regard to the evaluation of lung transplantation.

Received 22 ${ }^{\text {nd }}$ February 2009, accepted for publication 21 ${ }^{\text {st }}$ November 2009

${ }^{*}$ Equal contributors
However, existing models are hampered by various problems. Many researchers have used small animals like rabbits or rats, but the size and geometry of the murine lung is not comparable to human conditions. However, large animals are expensive and ethical standards require reduction of experimental animal numbers. In addition, existing perfusion models have employed synthetic perfusion solution.

Circumventing these concerns, we developed a porcine perfused isolated lung model based on already existing perfusion systems (Dittrich et al., 1998; Grosse-Siestrup et al., 2002; Modersohn et al., 2001) that uses organs from an abattoir. In order to gain unique possibilities for pharmacology and toxicology studies and improve graft function after transplantation, we perfused the lungs normo-thermically with autologous blood.

The aim of the current study was to evaluate the established model and to assess whether a stable organ function might be maintained in this model. 


\section{Animals}

To validate the model, a total of 6 lungs of white German landrace pigs (age 6 months, weight $100 \pm 10 \mathrm{~kg}$ ) were used and harvested at a commercial abattoir with approval of the official veterinarian institutions. The animals were housed and bred under standard conditions according to the EC-Guidelines for the accommodation and care of animals used for scientific purposes (K 2525, 2007), and the guidelines were approved by the official veterinarian institutions. Veterinarian inspections were performed on a routine basis and any transmissible diseases excluded. All experimental animal procedures were approved by the State Animal Committee on Use and Care of Animals. Protocols were performed in accordance with the European Commission (Council Directive 93/119).

\section{Materials and methods}

Organ and blood harvesting

The animals were electrically stunned according to the slaughtering protocol of the abattoir. Afterwards the animals were desanguinated through the cervical vessels and the autologous blood $(2,500$ to $3,000 \mathrm{ml})$ was collected in a sterile metal basin. The blood was anticoagulated with sodium citrate $(50 \mathrm{ml} / \mathrm{l})$ and heparin (10,000 IU/1, Liquemin, Hoffman-La Roche), filtered using a transfusion device, and stored in blood bags.

After blood harvesting, the chest was explored, the pleura was obtusely opened, and both lungs and the heart were removed by en bloc technique. The pulmonary trunk was then cannulated and flushed with the preservation solution LPD (low potassium dextrane, $2,000 \mathrm{ml}$, respectively at $4^{\circ} \mathrm{C}$ ). The trachea was intubated and the lung was inflated by a portable respirator with a constant pressure at 10 to $15 \mathrm{~cm} \mathrm{H}_{2} 0$.

The organs were then stored under sterile conditions at $4^{\circ} \mathrm{C}$, transferred to the laboratory, and re-perfused after a total cold storage time of $330 \pm 30$ minutes.

\section{Experimental Protocol}

A cannula was inserted before reperfusion of the organs into the pulmonary artery (pulmonary trunk was already cannulated at the abattoir). The organs were then transferred to water bed (containing $\mathrm{NaCl} 0.9 \%$ ), which was thermically adjusted to $37^{\circ} \mathrm{C}$ and rested on a balance system for gravimetric analysis (Sartorius, Göttingen, Germany) to control the blood volume and dilution.

The organ perfusion apparatus (Fig. 1) consisted of two separate circuits which are connected via a dialysis module (model F7, Fresenius, Bad Homburg, Germany). Based on the principal of reverse flow, the dialysis module allows diffusion of solute, fluid, and heat among the blood and the dialysis circuit. Circulation was established by two occlusion roller pumps (Stöckert, Munich, Germany). The circulating blood reached the membrane-oxygenator (COBE VPCML Plus, Cardiovascular inc., USA), which allowed a desoxygenation and carboxylation ( $79 \% \mathrm{~N}_{2} ; 20 \% \mathrm{CO}_{2}$ ). Powered by another roller pump, the blood passed an air-entrapment and sampling point (pressure, temperature, histological samples) and reached the pulmonary artery in

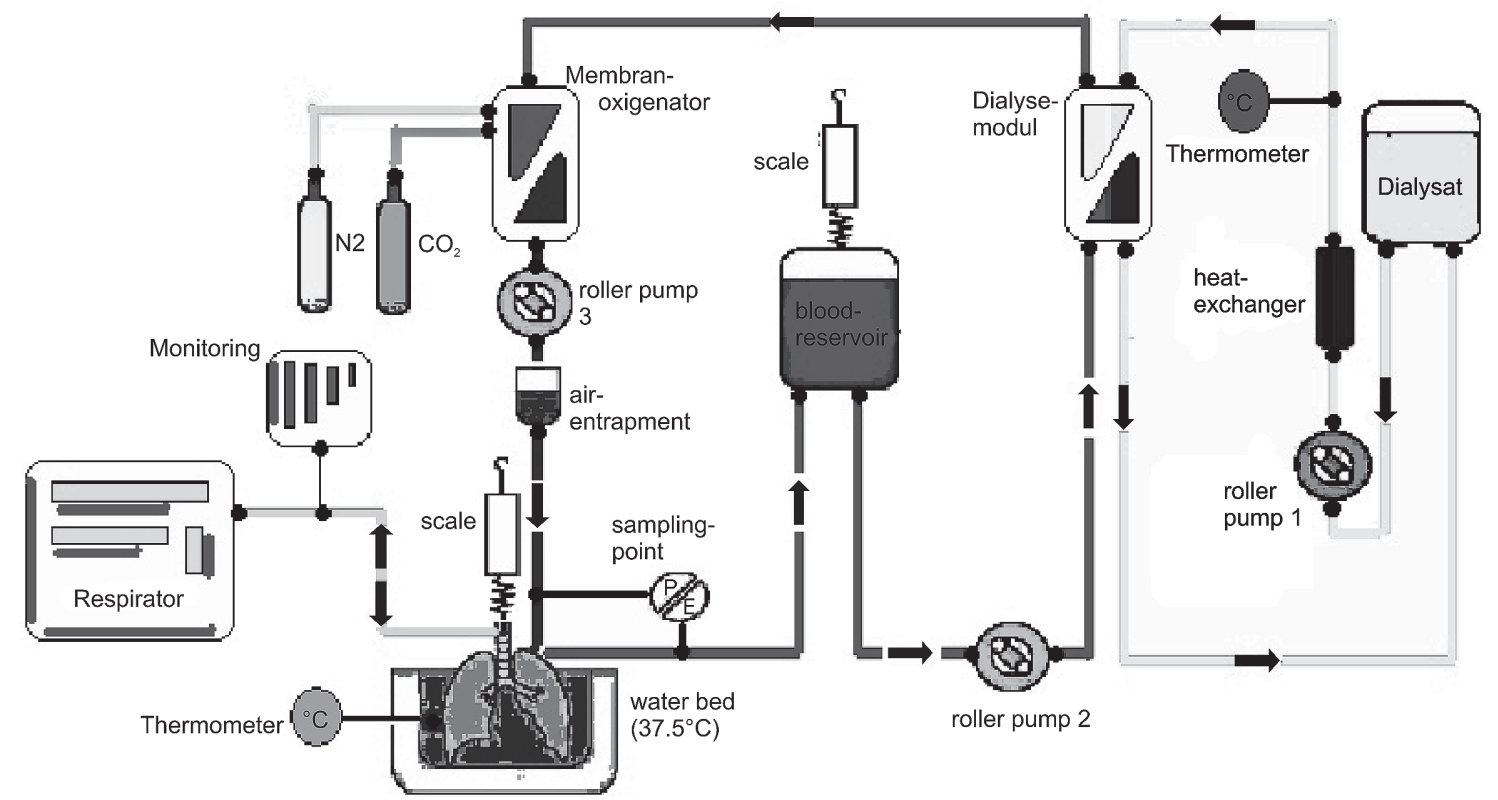

Fig. 1: Organ perfusion apparatus 
the lung. The last roller pump $(0.5 \mathrm{l} / \mathrm{min})$ offered regulation for constant perfusion flow. Blood passage, through a blood reservoir and finally the dialysis module, closed the circuit in the dialysis module. The dialysis circuit comprised a heat exchanger and the flow $(2 \mathrm{l} / \mathrm{min})$ was regulated by a roller pump.

The lung was connected to a respirator and the airflow was monitored (Servo Ventilator 900D, Siemens Elema, Sweden).

The respiration rate was adjusted for all attempts to 16 [1/min] with a breath minute volume of $7 \pm 0.1[1 / \mathrm{min}]$ and to a quotient expiration to inspiration of 0.33 . With the start of the perfusion respirated with an inspiratory oxygen concentration $\left(\mathrm{FiO}_{2}\right)$ of at first $100 \%$ afterwards of $21 \%$. This value was maintained up to the end of the perfusion. The pulmonale endexpiratory pressure (PEEP) amounted to $0.8[\mathrm{kPa}]$.

\section{Preparation of the perfusion model}

The protocol consisted of three stages: perfusion preparation, warm rinsing of the organ, and normo-thermic hemoperfusion. Initially, the dialysis circuit was filled with 3 liters of the fluid, which was adjusted to $37^{\circ} \mathrm{C}$. Afterwards the perfusion circuit was filled with heparinized physiological saline solution to avoid blood clotting. Simultaneously, the lung was prepared for the connection. The LPD (low potassium dextran) -preservation solution was washed out with isotonic saline solution and afterwards with blood through the pulmonary trunk ("warm rinsing"). A blood bag with $500 \mathrm{ml}$ was integrated into the circuit. The circuit was then closed and the blood passed the dialysis module and the membrane oxygenator.

On the side of the pulmonal artery the following values were adjusted: a $\mathrm{pH}$ value of $7.3 \pm 0.02$, a hemoglobin concentration $(\mathrm{tHb})$ of $10 \pm 0.2[\mathrm{~g} / \mathrm{l}]$, an oxygen partial pressure $\left(\mathrm{pO}_{2}\right)$ of 53 \pm 0.9 [mmHg], an oxygen saturation $\left(\mathrm{sO}_{2}\right)$ of $76 \pm 1.4$ [\%], and a carbon dioxide partial pressure $\left(\mathrm{pCO}_{2}\right)$ of $60 \pm 4.2[\mathrm{mmHg}]$. Also a sodium ion concentration of $144 \pm 0.5$ [mmol/l], a potassium ion concentration of $4.15 \pm 0.1$ [mmol/1] , and a calcium ion concentration of $1.09 \pm 0.1[\mathrm{mmol} / \mathrm{l}]$.

The normo-thermic hemoperfusion started. The point in time where the circulation was closed marked the beginning of the perfusion. Time was appointed to 135 minutes. Starting with $200 \mathrm{ml} / \mathrm{min}$ the blood flow then was raised step by step $(50 \mathrm{ml} /$ $\mathrm{min}$ ) until $500 \mathrm{ml} / \mathrm{min}$ in period of 45 minutes. A pulmonalarterial pressure $3.3 \mathrm{kPa}$ was aspired. An interval of 15 minutes for measuring data was adopted. After an average of 45 minutes a steady state of the organ performance was reached.

\section{Solutions}

The dialysis solution consisted of $\mathrm{Na}^{+} 130.5 \mathrm{mmol} / 1, \mathrm{P}^{+} 4 \mathrm{mmol} / \mathrm{l}$, $\mathrm{Mg}^{2+} 1 \mathrm{mmol} / 1, \mathrm{Cl}^{-} 115 \mathrm{mmol} / \mathrm{l}, \mathrm{Ca}^{2+} 1.5 \mathrm{mmol} / \mathrm{l}, \mathrm{H}_{2} \mathrm{PO}_{4}{ }^{-} 0.5$ $\mathrm{mmol} / \mathrm{l}, \mathrm{HCO}_{3} 20 \mathrm{mmol} / \mathrm{l}$, Glucose $8 \%$, Urea $60 \mathrm{mmol} / \mathrm{l}$, and Kreatinin $113 \mathrm{mmol} / \mathrm{l}$. The solution was freshly prepared for every run and was kept at $4^{\circ} \mathrm{C}$.

Preservation solution: As preservation solution low potassium dextran (LPD) was used containing $\mathrm{Na}^{+} 138 \mathrm{mmol} / \mathrm{l}$, $\mathrm{P}^{+} 6 \mathrm{mmol} / 1, \mathrm{Mg}^{2+} 0.8 \mathrm{mmol} / \mathrm{l}, \mathrm{Cl}^{-} 142 \mathrm{mmol} / 1, \mathrm{SO}_{4}{ }^{2-} 0.8 \mathrm{mmol} / \mathrm{l}$, $\mathrm{HPO}_{4}{ }^{2-} 0.3 \mathrm{mmol} / 1, \mathrm{H}_{2} \mathrm{PO}_{4}{ }^{-} 0.5 \mathrm{mmol} / 1$, dextran $5 \%$, Glucose $8 \%$, and Urea.

\section{Parameters}

Besides different hemodynamic parameters, numerous further parameters of organ viability were examined at the time points $0,15,30,45,60,75,90,105,120$, and $135 \mathrm{~min}$. Parameters measured included: for respiratory, the positive inspiratory pressure (PIP); for hemodynamics, the pulmonary arterial pressure (PAP); and for blood gases, the pulmonary vein oxygen partial pressure $\left(\mathrm{pO}_{2}\right)$. Lung weights were analyzed.

\section{Statistics}

Data are expressed as mean \pm standard deviation (SD). For the computation of the non-parametric tests, the SPSS version 11.0 program was used. For the comparison of independent samples, the Mann-Whitney-U test was selected, and for the comparison of dependent samples, the Wilcoxon test; $p$ values $<0.05$ were considered to represent statistical significance.

\section{Results}

\section{Respiratory mechanics}

For the evaluation of respiratory parameters, the positive inspiratory pressure (PIP), compliance, and the dynamic compliance were analyzed [Formula: tidal volume / PIP-PEEP].

A steady increase of the PIP was observed (15 minutes 20.43 \pm 1.74 [mbar]; 90 minutes $22.72 \pm 1.79$ [mbar]; and 135 minutes $25.23 \pm 1.66$ [mbar]) (Fig. 2). Significances are shown: $15 \mathrm{~min}$ to $90 \mathrm{~min}=0.045(*) ; 15 \mathrm{~min}$ to $135 \mathrm{~min}=0.028(*) ; 90 \mathrm{~min}$ to $135 \min =0.028(*)$.

\section{Hemodynamics}

Blood flow, arterial blood pressure, venous blood pressure, and capillary closing pressure were measured.

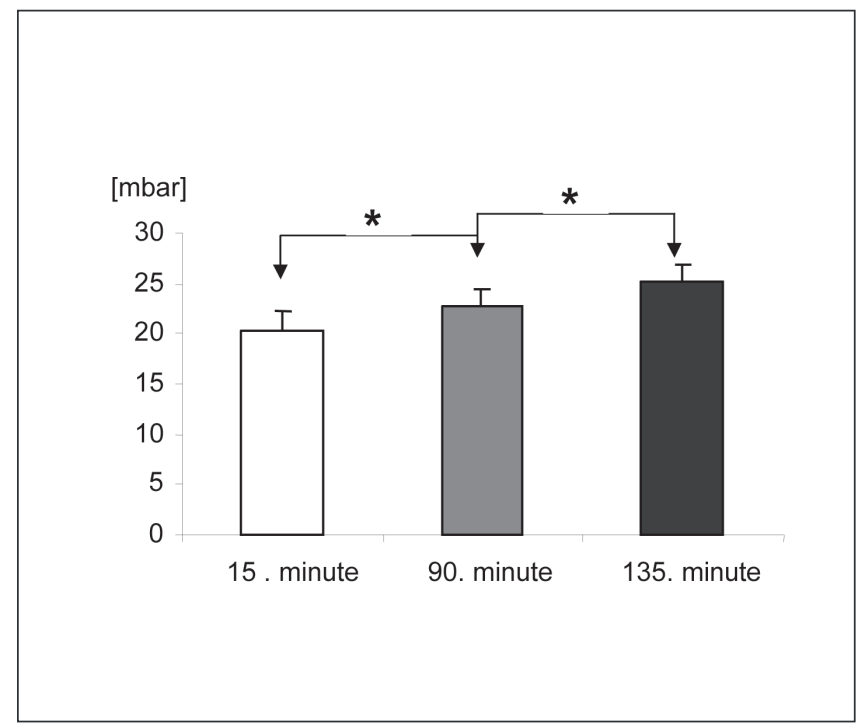

Fig. 2: Positive inspiratory pressure

The positive inspiratory pressure (PIP) was determined [mbar] in a porcine model of isolated and perfused lung at 3 time points (15, 90, $135 \mathrm{~min})$. A steady increase of PIP was noted. Significances are shown with ${ }^{*}(p<0.05)$. 
Pulmonary arterial pressure (PAP) was determined that reflected hemodynamic characteristics of the system. After reaching a steady state, the PAP remained stable over the examination period (15 minutes $19.83 \pm 1.3[\mathrm{mmHg} /(\mathrm{ml} / \mathrm{min} / \mathrm{g})] ; 90$ minutes $19.67 \pm 2.36[\mathrm{mmHg} /(\mathrm{ml} / \mathrm{min} / \mathrm{g})] ; 20 \pm 2.05[\mathrm{mmHg} /$ (ml/min/g)]) (Fig. 3).

\section{Blood gases}

Blood gases-parameters like pulmonary vein oxygen partial pressure, pulmonary vein carbon dioxide partial pressure, pulmonary vein oxygen saturation, pulmonary artery oxygen partial pressure, pulmonary artery carbon dioxide partial pressure, and pulmonary artery oxygen saturation were analyzed.

Pulmonary vein oxygen partial pressure $\left(\mathrm{pO}_{2}\right)$ : A steady decrease of the $\mathrm{pO}_{2}$ (Fig. 4) was measured: 15 minutes 494.67 \pm 62.39 [mmHg]; 90 minutes $72.3 \pm 4.22[\mathrm{mmHg}] ; 55.52 \pm 4.52$ $[\mathrm{mmHg}]$. Significances are shown: 15 to $90 \mathrm{~min}=0.028(*) ; 15$ to $135 \mathrm{~min}=0.028(*) ; 90$ to $135 \mathrm{~min}=0.027(*)$.

Lung weight

The relative lung weight was calculated [Formula: (LG perf -LG nat)/LG nat)*10]. Basic value was the gravimetric analysis after 15 minutes hemoperfusion. In relation to the data after 90 minutes (beginning of the steady-state) and after 135 minutes (final measuring), the relative lung weight was calculated.

An increase in lung weight (Fig. 5) was determined: after 90 minutes about $13.62 \pm 3.63[\%]$; after 135 minutes 16.32 $\pm 4.78[\%]$.

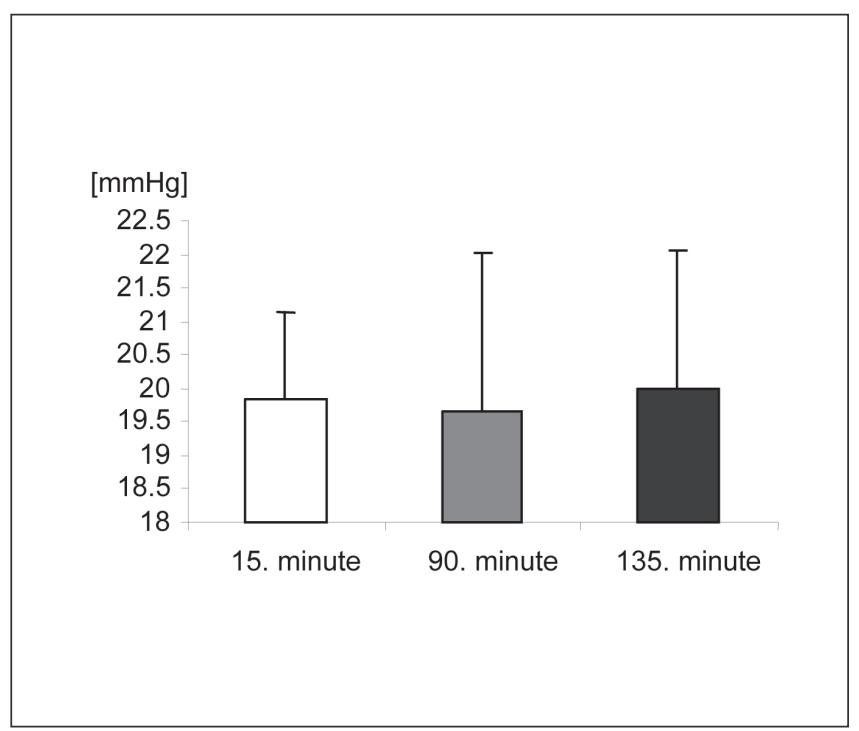

Fig. 3: Pulmonary arterial pressure The pulmonary arterial pressure (PAP) was determined in [mmHg] in a porcine model of isolated and perfused lung at 3 time points (15, 90, $135 \mathrm{~min})$.

\section{Discussion}

In the present paper we report on the evaluation of a new model of isolated, autologously hemoperfused porcine slaughterhouse lungs. In contrast to previously models (Fehrenbach et al., 1999), this approach takes advantages of the close physiological proximity between porcine species, as donor species, and human organs. Also, the use of slaughterhouse organs reduces the number of laboratory animals.

A number of studies have used rodents such as rats or rabbits as donor species in models of isolated perfused lungs to examine pulmonary functions (Ghofrani et al., 2001; Lockinger et al., 2001). However, these animals display significant differences in organ size and geometry as compared to humans. Hammer et al. declared that the organ size, the anatomical and physiological characteristics of the human and porcine lung are all about the same (Hammer et al., 1998). In this regard, pig lungs used in the current model present a better approach to reproduce in vivo conditions found in humans.

The model uses a whole organ approach, which preserves the three-dimensional organ structure with its cell-to-cell interactions. In contrast to precision cut lung slices (Ressmeyer et al., 2006) or cultured lung cells (Boekema et al., 2003; Jung et al., 2004), it allows the assessment of a large range of whole organ functions. Analyzing these, we found indications of an ischemic lung injury. The positive inspiratory pressure (PIP) represents the airway pressure at the end of inspiration and might be used as parameter evaluating airway function (Fehrenbach et al., 1999). This parameter is affected by a variety of variables, e.g. the airway resistance and compliance. A decrease in compliance caused by pathological phenomena in the lung (e.g. edema, pneumonia, and atelectasis) results in

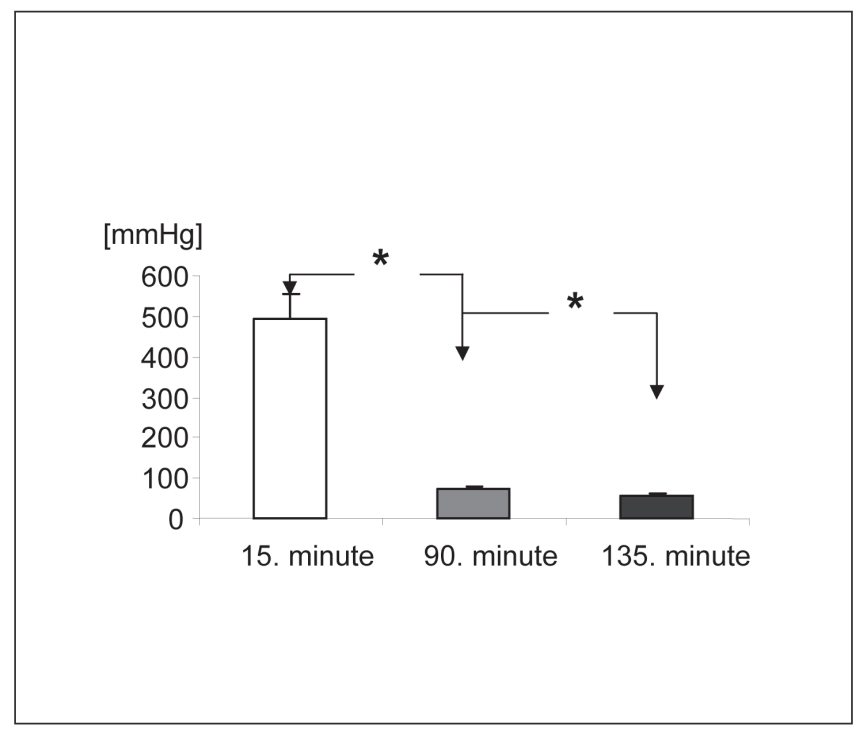

Fig. 4: Pulmonary vein oxygen partial pressure Pulmonary vein oxygen partial pressure $\left(\mathrm{pO}_{2}\right)$ was determined $[\mathrm{mmHg}]$ in a porcine model of isolated and perfused lung at 3 time points $(15,90,135 \mathrm{~min})$. A steady decrease in $\mathrm{pO}_{2}$ was noted. Significances are shown with * $(p<0.05)$. 
an increase in PIP as well as a rise in airway resistance (e.g. broncho-spasm) (Yanes et al., 1971). The increase in PIP seen during our study can be mostly attributed to the development of lung edema. To this end, microscopic studies in a similar model by Fehrenbach and co-worker showed that the PIP is correlated with the intra-alveolar edema (Fehrenbach et al., 1999). Also, we found an increasing lung weight, which is the expression of lung edema caused by a post-ischemic reperfusion injury. Elevated capillary pressure and permeability play an important role in the post-ischemic process (West et al., 1991). During this process an interstitial edema that develops beside the alveolar edema causes diffusion impairment and a decreased oxygen uptake (Fehrenbach et al., 2001). In the present model pulmonary vein oxygen partial pressure $\left(\mathrm{pvO}_{2}\right)$ was a measure for the gas exchange. By controlling other factors responsible for a decreased gas transport (like ventilation and changes in perfusion), a diffusion impairment caused by edema is most likely. This is in line with the observation of other groups that found that the ischemic reperfusion injury is characterized by reduced $\mathrm{pvO}_{2}$ (Kemming et al., 1998).

The pulmonary arterial pressure (PAP) is regulated by the muscular tone of the blood vessels. Damage of the endothelium results in the liberation of mediators. Excess of vasoconstrictive mediators will result in a constriction of the blood vessels. The pulmonary arterial pressure will increase (Grace, 1994; Novick et al., 1996). Concerning the PAP, there were no changes noted over a period of 135 minutes; therefore, we might rule out major damage of the endothelium for the operative procedure.

Ischemic reperfusion injury with similar changes in airway parameters is often seen in patients that have been lung transplanted; therefore, the model might specially serve in studying this subject.

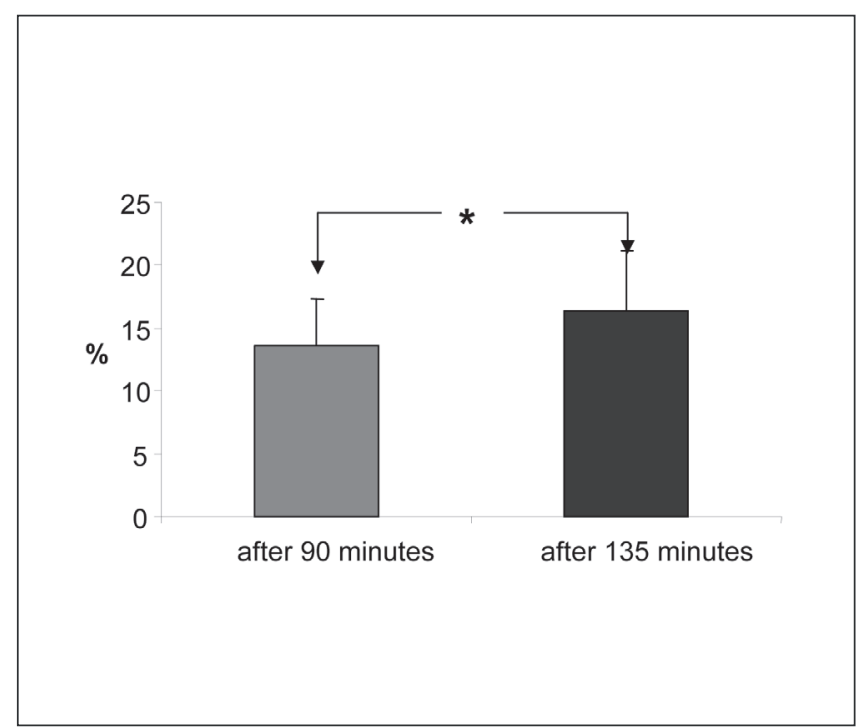

Fig. 5: Relative lung weight increase

The relative lung weight was determined [\%] in a porcine model of isolated and perfused lung at 2 time points (90 and $135 \mathrm{~min}$ ). An increase of the lung weight was noted. Significances are shown with * $(p<0.05)$.
Laboratory animal experiments are increasingly used, especially for biological and medical research (Taylor et al., 2008). Recent estimates of worldwide annual laboratory animal use range from 28-100 million.

The current model uses slaughterhouse organs. In particular, for pathophysiological questions in medicine, this method represents an alternative and the number of laboratory animals can be reduced.

In accordance to the official rules of the slaughtering process, the lungs were harvested at an abattoir and transferred to the laboratory. In view oft he stable values obtained in the study, slaughterhouse lungs therefore may be used efficiently. Legally, slaughterhouse lung perfusion studies are not defined as animal experiments and therefore fulfill international standards in terms of establishing alternatives to animal experimentation

In summary, the present study was carried out to establish a new isolated hemoperfused model that approximates human lung physiology. The use of slaughterhouse porcine lungs and autologous blood reduces the number of laboratory animals necessary for studies. The model might be used in future investigations to understand the pathophysiology of the ischemic-reperfusion damage and influence the underlying pathophysiological processes. Also, toxicological questions and studies about organ preservation might be done with this model to further reduce laboratory animal use.

\section{References}

Boekema, B. K., Stockhofe-Zurwieden, N., Smith, H. E. et al. (2003). Adherence of Actinobacillus pleuropneumoniae to primary cultures of porcine lung epithelial cells. Vet. Microbiol. 93, 133-144.

Dittrich, S., Schuth, A., von Baeyer, H. et al. (1998). [Effect of blood viscosity on the function of isolated perfused porcine kidney after cold preservation]. Zentralbl. Chir. 123, 809813.

EC Council Directive 93/119/ EC Official Journal L340 31/12/193 p.0021-0034

EC-Guidelines (2007). K 2525

Fehrenbach, A., Fehrenbach H., Wittwer, T. et al. (2001). Evaluation of pulmonary edema: stereological versus gravimetrical analysis. Eur. Surg. Res. 33, 270-278.

Fehrenbach, H., Schepelmann, D., Albes, J. M. et al. (1999). Pulmonary ischemia/reperfusion injury: a quantitative study of structure and function in isolated heart-lungs of the rat. Anat. Rec. 255, 84-89.

Ghofrani, H. A., Kohstall, M. G., Weissmann, N. et al. (2001). Alveolar epithelial barrier functions in ventilated perfused rabbit lungs. Am. J. Physiol. Lung Cell Mol. Physiol. 280, L896-904.

Grace, P. A. (1994). Ischaemia-reperfusion injury. The British Journal of Surgery 81, 637-647.

Grosse-Siestrup, C., Fehrenberg, C., von Baeyer, H. and Groneberg, D. A. (2002). Multiple-organ harvesting for models of isolated hemoperfused organs of slaughtered pigs. ALTEX 19, 9-13.

Hammer, C., Linke, R., Wagner, F. and Diefenbach, M. (1998). 
Organs from animals for man. Int. Arch. Allergy Immunol. $116,5-21$.

Jung, K., Ha, Y., Ha, S. K. et al. (2004). Antiviral effect of Saccharomyces cerevisiae beta-glucan to swine influenza virus by increased production of interferon-gamma and nitric oxide. J. Vet. Med. B Infect. Dis. Vet. Public Health 51, 72-76.

Kemming, G. I., Merkel, M. J., Schallerer, A.et al. (1998). Inhaled nitric oxide (NO) for the treatment of early allograft failure after lung transplantation. Munich Lung Transplant Group. Intensive Care Med.24, 1173-1180.

Kimose, H. H., Ravkilde, J., Knudsen, M. A. et al. (1990). Recovery after cold cardioplegic arrest of isolated blood-perfused hearts excised from non-anesthetized pigs. Eur. Surg. Res. 22, 323-335.

Lockinger, A., Schutte, H., Walmrath, D. et al. (2001). Protection against gas exchange abnormalities by pre-aerosolized PGE1, iloprost and nitroprusside in lung ischemia-reperfusion. Transplantation 71, 185-193.

Modersohn, D., Eddicks, S., Grosse-Siestrup, C. et al. (2001). Isolated hemoperfused heart model of slaughterhouse pigs. Int. J. Artif. Organs 24, 215-221.

Novick, R. J., Gehman, K. E., Ali, I. S. and Lee, J. (1996). Lung preservation: the importance of endothelial and alveolar type II cell integrity. Ann. Thorac. Surg. 62, 302-314.

Ressmeyer, A. R., Larsson, A. K., Vollmer, E. et al. (2006). Characterisation of guinea pig prescsion-cut lung slices comparsion with human tissue. Eur. Respir. J. 28, 603-611.

Samaja, M., Rovida, E., Motterlini, R. et al. (1990). Human red cell age, oxygen affinity and oxygen transport. Respir. Physiol. 79, 69-79.

Schneuwly, O. D., Licker, M., Pastor, C. M. et al. (1999). Beneficial effects of leukocyte-depleted blood and low-potassium dextran solutions on microvascular permeability in preserved porcine lung. Am. J. Respir. Crit. Care Med. 160, 689-697.

Taylor, K., Gordon, N., Langley, G. and Higgins, W. (2008). Estimates for worldwide laboratory animal use in 2005. ATLA 36, 327-342.

West, J. B., Tsukimoto, K., Mathieu-Costello, O. et al. (1991). Stress failure in pulmonary capillaries. J. Appl. Physiol. 70, 1731-1742.

Wright, N. C., Hopkinson, D. N., Shaw, T. E. et al. (2000). A porcine ex vivo paracorporeal model of lung transplantation. Lab. Anim. 34, 56-62.

Yanes, H. O., Rudy, L. W., Wagner, O. A. et al. (1971). Pulmonary arterial blood flow to transplanted lungs of standing dogs. Surg. Forum 22, 218-221.

\section{Correspondence to}

Peter C. Kamusella, MD

Department of Radiology

Westküstenkliniken Heide

Esmarchstr. 50

25746 Heide

Germany

e-mail: paipman@web.de 